\title{
SIMPLE WEIGHT MODULES OVER WEAK GENERALIZED WEYL ALGEBRAS
}

\author{
RENCAI LÜ, VOLODYMYR MAZORCHUK AND KAIMING ZHAO
}

\begin{abstract}
In this paper we address the problem of classification of simple weight modules over weak generalized Weyl algebras of rank one. The principal difference between weak generalized Weyl algebras and generalized weight algebras is that weak generalized Weyl algebras are defined using an endomorphism rather than an automorphism of a commutative ring $R$. We reduce classification of simple weight modules over weak generalized Weyl algebras to description of the dynamics of the action of the above mentioned endomorphism on the set of maximal ideals. We also describe applications of our results to the study of generalized Heisenberg algebras.
\end{abstract}

\section{IntRoduction AND DESCRIPTION OF THE RESUlts}

Let $R$ be a commutative unital ring, $\sigma: R \rightarrow R$ an automorphism and $t \in R$. With the datum $(R, \sigma, t)$ one associates a generalized Weyl algebra $A=A(R, \sigma, t)$ of rank one defined as an $R$-algebra generated by elements $X$ and $Y$ subject to the relations

(1.1) $Y X=t, X Y=\sigma(t), X r=\sigma(r) X, r Y=Y \sigma(r)$ for all $r \in R$.

This class of algebras was introduced by Bavula in [Ba1] and later investigated by various authors, see, in particular, Ba2, BJ, DGO, Maz, Sh, Ha2 and references therein. Generalized Weyl algebras were further studied and generalized to natural higher rank analogues, see for example [BB, $\mathrm{MT2}, \mathrm{BO}]$, and then to certain twisted and multi parameter versions, see for example [MT1, MT3, Ha1, FH1, FH2] and references therein. Many more papers studying generalized Weyl algebras can be found using Google search or searching MathSciNet.

One of the main reasons why these algebras attracted such a considerable attention is that the class of generalized Weyl algebras contains many interesting algebras. Thus the first Weyl algebra, the universal enveloping algebra of $\mathfrak{s l}_{2}$, the quantum algebra $U_{q}\left(\mathfrak{s l}_{2}\right)$ and most of the down-up algebras from [BR] are all examples of generalized Weyl

Date: July 24, 2018

Keywords: algebra; category; module; weight module 2010 Math. Subj. Class.: 
algebras of rank one. Higher rank, twisted and multi parameter generalizations cover much more, we refer the reader to the corresponding papers for examples.

One common feature of all studies mentioned above is that the datum defining a generalized Weyl algebra (or its generalization) consists of a base ring (usually commutative and unital), a collection of automorphisms of this ring, a collection of elements of this ring and, maybe, some more stuff. At the same time, the relations (1.1) make perfect sense for any endomorphism $\sigma$ of $R$ (which we always assume to be unital, that is $\sigma(1)=1$ ). To distinguish the latter case from the original generalized Weyl algebras we will add the word weak to the classical name of the algebra and abbreviate weak generalized Weyl algebras as $w G W A$.

Weakening the requirement for $\sigma$ from being an automorphism to being an endomorphism leads to a new family of interesting examples, namely the so-called generalized Heisenberg algebras, see [CRM, LZ], defined as follows: Let $f(h) \in \mathbb{C}[h]$ be a fixed polynomial. Then the corresponding generalized Heisenberg algebra $\mathcal{H}(f)$ is defined as the $\mathbb{C}$-algebra generated by $X, Y$ and $h$ subject to the following relations:

$$
h Y=Y f(h) ; \quad X h=f(h) X ; \quad[X, Y]=f(h)-h .
$$

These algebras have many applications in theoretical physics, see the references in [LZ for details. To realize $\mathcal{H}(f)$ as a wGWA, we may, for example, take $R=\mathbb{C}[h, z]$, set $t=h+z$ and choose $\sigma$ as the endomorphism of $R$ sending $z$ to $z$ and $h$ to $f(h)$. It is immediate to check that, using the correspondence $X \leftrightarrow X, Y \leftrightarrow Y$ and $Y X \leftrightarrow h+z$, the defining relations (1.2) transfer into (1.1) and vise versa.

This naturally leads to the question of which results for generalized Weyl algebras can be extended to weak generalized Weyl algebras and how. The paper [LZ] addresses the problem of classification of simple finite dimensional modules over generalized Heisenberg algebra and makes some progress in study of simple weight modules. In this paper we use the ideas and methods from [DGO] to study weight modules for general weak generalized Weyl algebras. It turns out that there are several subtle differences between GWAs and wGWAs which should be treated with care. To emphasize these differences and to avoid unnecessary higher rank technical complications we restrict to the rank one case. This is still enough for our main application, which is generalized Heisenberg algebras. A nice and unusual feature of the algebras we consider is existence of simple weight modules with both non-trivial finite dimensional and infinite dimensional weight spaces, see Example 32. 
The paper is organized as follows: in Section 2 we collect some basic facts on simple weight modules over generalized Weyl algebras and specify our main restriction on the defining endomorphism, namely that we always assume that this endomorphism is essential, see Subsection 2.3. In Subsection 2.4 we define several families of weight modules which we call string modules. In Section 3 we establish the classical decomposition of the category of weight modules. In Section 4 we first describe the support of a simple weight module in Proposition 22 and then prove our main classification result: Theorem 27. In Section 5 we derive some corollaries about finite dimensional modules and, finally, in Section [6 we describe applications to generalized Heisenberg algebras.

Acknowledgements. R.L. is partially supported by NSF of China (Grant 11371134) and Jiangsu Government Scholarship for Overseas Studies (JS-2013-313).

V. M. is partially supported by the Swedish Research Council.

K.Z. is partially supported by NSF of China (Grant 11271109) and NSERC.

\section{Weight MODUles OVER WEAK GENERALIZED WeYL ALGEBRAS}

2.1. Weight and generalized weight modules. Let $A=A(R, \sigma, t)$ be a wGWA. Let $\operatorname{Max}(R)$ denote the set of all maximal ideals in $R$. For an $A$-module $M$ and $\mathbf{m} \in \operatorname{Max}(R)$ set

$$
M_{\mathbf{m}}:=\{v \in M: \mathbf{m} v=0\}, \quad M^{\mathbf{m}}:=\left\{v \in M: \mathbf{m}^{k} v=0, k \gg 0\right\} .
$$

A module $M$ is called a weight module provided that

$$
M=\bigoplus_{\mathbf{m} \in \operatorname{Max}(R)} M_{\mathbf{m}}
$$

A module $M$ is called a generalized weight module provided that

$$
M=\bigoplus_{\mathbf{m} \in \operatorname{Max}(R)} M^{\mathbf{m}} .
$$

Clearly, any weight module is a generalized weight module.

For a weight $A$-module $M$ define its support as the set

$$
\operatorname{supp}(M):=\left\{\mathbf{m} \in \operatorname{Max}(R): M_{\mathbf{m}} \neq 0\right\} .
$$

Similarly one defines the support of a generalized weight module.

For $\mathbf{m} \in \operatorname{Max}(R)$ we denote by $\mathbb{k}_{\mathbf{m}}$ the quotient field $R / \mathbf{m}$. 


\subsection{Action of generators on weight vectors.}

Lemma 1. Let $M$ be a weight $A$-module, $\mathbf{m} \in \operatorname{supp}(M)$ and $v \in M_{\mathbf{m}}$ be a nonzero element. Then $X v=0$ implies $t \in \mathbf{m}$. Furthermore, $Y v=0$ implies $\sigma(t) \in \mathbf{m}$.

Proof. From $X v=0$ we have $0=Y X v=t v$ using (1.1) which implies $t \in \mathbf{m}$. Similarly, from $Y v=0$ we have $0=X Y v=\sigma(t) v$ (again using (1.1)) which implies $\sigma(t) \in \mathbf{m}$.

Lemma 2. Let $M$ be a weight $A$-module and $\mathbf{m} \in \operatorname{supp}(M)$. Then

$$
X M_{\mathbf{m}} \subset \bigoplus_{\mathbf{n}: \sigma(\mathbf{m}) \subset \mathbf{n}} M_{\mathbf{n}}
$$

Proof. Let $v$ be a nonzero element in $M_{\mathbf{m}}$. Write $X v=w_{1}+w_{2}+\cdots+w_{k}$ where for each $i$ we have $\mathbf{n}_{i} w_{i}=0$ for some $\mathbf{n}_{i} \in \operatorname{Max}(R)$. Without loss of generality we may assume $w_{i} \neq 0$ for all $i$ and $\mathbf{n}_{i} \neq \mathbf{n}_{j}$ if $i \neq j$. Using (1.1) we have

$$
0=X \mathbf{m} v=\sigma(\mathbf{m}) X v=\sum_{i} \sigma(\mathbf{m}) w_{i}
$$

which means that $\sigma(\mathbf{m}) w_{i}=0$ and hence $\sigma(\mathbf{m}) \subset \mathbf{n}_{i}$ (for each $i$ ). The claim follows.

Lemma 3. Let $M$ be a weight $A$-module and $\mathbf{m} \in \operatorname{supp}(M)$. Assume that one of the following holds:

(a) We have $\sigma(t) \notin \mathbf{m}$.

(b) There is $\mathbf{n} \in \operatorname{supp}(M)$ such that $\sigma(\mathbf{n}) \subset \mathbf{m}$.

Then

$$
Y M_{\mathbf{m}} \subset \bigoplus_{\mathbf{n}: \sigma(\mathbf{n}) \subset \mathbf{m}} M_{\mathbf{n}}
$$

Proof. Let $v$ be a nonzero element in $M_{\mathbf{m}}$. Write $Y v=w_{1}+w_{2}+\cdots+w_{k}$ where $\mathbf{n}_{i} w_{i}=0$ for some $\mathbf{n}_{i} \in \operatorname{Max}(R)$ (as above, we assume $w_{i} \neq 0$ for all $i$ and $\mathbf{n}_{i} \neq \mathbf{n}_{j}$ if $i \neq j$ ). Using (1.1) we have

$$
0=\mathbf{n}_{1} \mathbf{n}_{2} \cdots \mathbf{n}_{k} Y v=Y \sigma\left(\mathbf{n}_{1} \mathbf{n}_{2} \cdots \mathbf{n}_{k}\right) v .
$$

If we have $\sigma(t) \notin \mathbf{m}$ (that is condition (国) is satisfied), then the action of $Y$ on $M_{\mathbf{m}}$ is injective by Lemma 1 and hence $\sigma\left(\mathbf{n}_{1} \mathbf{n}_{2} \cdots \mathbf{n}_{k}\right) v=0$, that is we have the inclusion $R \sigma\left(\mathbf{n}_{1} \mathbf{n}_{2} \cdots \mathbf{n}_{k}\right) R \subset \mathbf{m}$. Then we have

$$
R \sigma\left(\mathbf{n}_{1}\right) R R \sigma\left(\mathbf{n}_{2}\right) R \cdots R \sigma\left(\mathbf{n}_{k}\right) R \subset \mathbf{m} .
$$

As $\mathbf{m}$ is maximal (and hence prime), we get $R \sigma\left(\mathbf{n}_{i}\right) R \subset \mathbf{m}$ (which is equivalent to $\left.\sigma\left(\mathbf{n}_{i}\right) \subset \mathbf{m}\right)$ for some $i$. This shows that condition (b) is satisfied. 
Let us now prove the claim in the case when condition (b $\mathrm{b})$ is satisfied. If there is $\mathbf{n} \in \operatorname{supp}(M)$ such that $\sigma(\mathbf{n}) \subset \mathbf{m}$, then from (1.1) we have $\mathbf{n} Y v=Y \sigma(\mathbf{n}) v=0$, which implies $\mathbf{n}=\mathbf{n}_{i}$ for all $i$. The claim follows.

Corollary 4. Let $\mathbf{m} \in \operatorname{Max}(R)$ be such that $\sigma(t) \notin \mathbf{m}$ and for any $\mathbf{n} \in \operatorname{Max}(R)$ we have $\sigma(\mathbf{n}) \not \subset \mathbf{m}$. Then for any weight module $M$ we have $M_{\mathbf{m}}=0$.

Proof. Assume $v \in M_{\mathbf{m}}$ is nonzero. Then $\sigma(t) v \neq 0$ as $\sigma(t) \notin \mathbf{m}$ and hence $Y v \neq 0$ either. As for any $\mathbf{n} \in \operatorname{Max}(R)$ we have $\sigma(\mathbf{n}) \not \subset \mathbf{m}$, we get a contradiction with Lemma 3 .

Lemma 5. For any $\mathbf{m} \in \operatorname{Max}(R)$ there is at most one $\mathbf{n} \in \operatorname{Max}(R)$ such that $\sigma(\mathbf{n}) \subset \mathbf{m}$.

Proof. Assume $\mathbf{k}, \mathbf{n} \in \operatorname{Max}(R)$ are such that $\sigma(\mathbf{k}) \subset \mathbf{m}, \sigma(\mathbf{n}) \subset \mathbf{m}$ and $\mathbf{k} \neq \mathbf{n}$. Then $\mathbf{k}+\mathbf{n}=R$ and hence $\sigma(R)=\sigma(\mathbf{k}+\mathbf{n}) \subset \mathbf{m}$ which contradicts $\sigma(1)=1$ (recall that $\sigma$ was assumed to be a unital endomorphism of $R$ ).

2.3. Essential endomorphisms. We say that $\sigma$ is essential provided that for any $\mathbf{m} \in \operatorname{Max}(R)$ the canonical inclusion

$$
\sigma(R) /(\sigma(R) \cap \mathbf{m}) \hookrightarrow R / \mathbf{m}
$$

is surjective. Obviously, if $\sigma$ is an automorphism, then it is an essential endomorphism.

Example 6. Assume that $R$ is a finitely or countably generated algebra over an uncountable algebraically closed field $\mathbb{k}$ and $\sigma$ is $\mathbb{k}$-linear. Then $\mathbb{k}_{\mathbf{m}} \cong \mathbb{k}$ for any $\mathbf{m} \in \operatorname{Max}(R)$ (see for example, [FGM, Lemma 1]) and hence any (unital) endomorphism of $R$ is essential.

Example 7. Let $\sigma: \mathbb{C} \rightarrow \mathbb{C}$ be a non-surjective ring homomorphism. Then such $\sigma$ is obviously not essential. Existence of such $\sigma$ follows from the fact that the algebraic closure of the field $\mathbb{C}(x)$ of rational functions is isomorphic to $\mathbb{C}$ by Steinitz Theorem, see [St, Page 125]. As $\sigma$ we thus can take the composition $\mathbb{C} \subsetneq \mathbb{C}(x) \subsetneq \overline{\mathbb{C}(x)} \cong \mathbb{C}$.

From now on we always assume that $\sigma$ is essential.

Our main interest in essential morphisms is the following property which we will use frequently:

Lemma 8. Assume that $\sigma$ is essential. Let $M$ be a weight A-module, $\mathbf{m} \in \operatorname{supp}(M)$ and $v \in M_{\mathbf{m}}$. Then $R v=\sigma(R) v$.

Proof. We have $R v=\mathbb{k}_{\mathbf{m}} v$ since $\mathbf{m} v=0$. Further, $\mathbb{k}_{\mathbf{m}} v=\sigma(R) v$ since $\sigma$ is essential. 
Further, for essential $\sigma$ we can strengthen Lemma 3 as follows:

Lemma 9. Assume that $\sigma$ is essential. Let $M$ be a weight A-module, $\mathbf{m} \in \operatorname{supp}(M)$ and assume that $Y M_{\mathbf{m}} \neq 0$. Then there is a unique $\mathbf{n} \in \operatorname{supp}(M)$ such that $\sigma(\mathbf{n}) \subset \mathbf{m}$, moreover, $Y M_{\mathbf{m}} \subset M_{\mathbf{n}}$.

Proof. Taking into account Lemmata 3 and 5, we only need to prove the existence of $\mathbf{n} \in \operatorname{supp}(M)$ such that $\sigma(\mathbf{n}) \subset \mathbf{m}$.

Let $v$ be a nonzero element in $M_{\mathbf{m}}$ such that $Y v \neq 0$. Write $Y v=$ $w_{1}+w_{2}+\cdots+w_{k}$ where $\mathbf{n}_{i} w_{i}=0$ for some $\mathbf{n}_{i} \in \operatorname{Max}(R)$. We assume $w_{i} \neq 0$ for all $i$ and $\mathbf{n}_{i} \neq \mathbf{n}_{j}$ if $i \neq j$. On the one hand, $\mathbf{n}_{k} Y v=Y \sigma\left(\mathbf{n}_{k}\right) v$ by (1.1). On the other hand,

$$
\mathbf{n}_{k} Y v=\mathbf{n}_{k}\left(w_{1}+w_{2}+\cdots+w_{k}\right)=\mathbf{n}_{k} w_{1}+\mathbf{n}_{k} w_{2}+\cdots+\mathbf{n}_{k} w_{k-1}
$$

since $\mathbf{n}_{k} w_{k}=0$. Moreover, $\mathbf{n}_{k} w_{i} \neq 0$ for all $i<k$ since $\mathbf{n}_{k} \neq \mathbf{n}_{i}$. Therefore we may assume $k=1$ and set $w:=w_{1}$ and $\mathbf{n}:=\mathbf{n}_{1}$.

Assume $\sigma(\mathbf{n}) \not \subset \mathbf{m}$. We have $0=\mathbf{n} Y v=Y \sigma(\mathbf{n}) v$. Pick $a \in \mathbf{n}$ such that $\sigma(a) \notin \mathbf{m}$. As $\sigma$ is essential, there is $b \in R$ such that

$$
(\sigma(b)+\mathbf{m})(\sigma(a)+\mathbf{m})=1+\mathbf{m} .
$$

On the one hand, we have $b a Y v=0$ since $a Y v=0$. On the other hand, by (1.1) we have

$$
b a Y v=Y(\sigma(b) \sigma(a) v) \stackrel{(2.1)}{=} Y(1 v)=Y v \neq 0
$$

by our assumptions, a contradiction. This completes the proof.

2.4. String modules. Denote by $\infty_{\infty} Q_{\infty}$ the set of all possible maps $\mathbb{Z} \rightarrow \max (R), i \mapsto \mathbf{m}_{i}$, such that $\sigma\left(\mathbf{m}_{i}\right) \subset \mathbf{m}_{i+1}$ for all $i \in \mathbb{Z}$. We will write $\underline{\mathbf{m}}:=\left(\mathbf{m}_{i}\right) \in{ }_{\infty} Q_{\infty}$ if the collection $\left(\mathbf{m}_{i}\right)$ comes from such a map.

For $i \in \mathbb{Z}$ define $X: \mathbb{k}_{\mathbf{m}_{i}} \rightarrow \mathbb{k}_{\mathbf{m}_{i+1}}$ as mapping $r+\mathbf{m}_{i}$ to $\sigma(t r)+\mathbf{m}_{i+1}$. Further, define $Y: \mathbb{k}_{\mathbf{m}_{i}} \rightarrow \mathbb{k}_{\mathbf{m}_{i-1}}$ as mapping $r+\mathbf{m}_{i}$ to $s+\mathbf{m}_{i-1}$ for some $s \in R$ such that $\sigma(s) \in r+\mathbf{m}_{i}$. Such an $s \in R$ exists because $\sigma$ is essential. For $\underline{\mathbf{m}} \in{ }_{\infty} Q_{\infty}$ set

$$
N(\underline{\mathbf{m}}):=\bigoplus_{i \in \mathbb{Z}} \mathbb{k}_{\mathbf{m}_{i}}
$$

Then $N(\underline{\mathbf{m}})$ carries the natural structure of an $R$-module. Note that, by construction, the action of $Y$ on $N(\underline{\mathbf{m}})$ defined above is bijective.

Lemma 10. The above defines on $N(\underline{\mathbf{m}})$ the structure of a weight $A$ module. 
Proof. First let us check that the action of $Y$ is well-defined. If $s, s^{\prime} \in R$ are such that $\sigma(s), \sigma\left(s^{\prime}\right) \in r+\mathbf{m}_{i}$, then $\sigma\left(s-s^{\prime}\right) \in \mathbf{m}_{i}$. Since $\sigma$ is unital, in particular, nonzero and $\mathbb{k}_{\mathbf{m}_{i-1}}$ is a field, it follows that $s-s^{\prime} \in \mathbf{m}_{i-1}$. Therefore the action of $Y$ is well-defined.

It remains to check relations (1.1). For $r \in R$ and $i \in \mathbb{Z}$ we have (using the notation above)

$$
\begin{gathered}
X Y\left(r+\mathbf{m}_{i}\right)=X\left(s+\mathbf{m}_{i-1}\right)=\sigma(t s)+\mathbf{m}_{i}=\sigma(t) r+\mathbf{m}_{i}, \\
Y X\left(r+\mathbf{m}_{i}\right)=Y\left(\sigma(t r)+\mathbf{m}_{i+1}\right)=t r+\mathbf{m}_{i} .
\end{gathered}
$$

Moreover, for $a \in R$ we also have

$$
\begin{aligned}
X a\left(r+\mathbf{m}_{i}\right)=X\left(a r+\mathbf{m}_{i}\right) & =\sigma(t a r)+\mathbf{m}_{i+1}= \\
= & \sigma(a)\left(\sigma(t r)+\mathbf{m}_{i+1}\right)=\sigma(a) X\left(r+\mathbf{m}_{i}\right) .
\end{aligned}
$$

Finally, for $a \in R$ we have

$$
a Y\left(r+\mathbf{m}_{i}\right)=a\left(s+\mathbf{m}_{i-1}\right)=a s+\mathbf{m}_{i-1}
$$

and

$$
Y \sigma(a)\left(r+\mathbf{m}_{i}\right)=Y\left(\sigma(a) r+\mathbf{m}_{i}\right)=s^{\prime}+\mathbf{m}_{i-1},
$$

where $s^{\prime}$ is such that $\sigma\left(s^{\prime}\right)+\mathbf{m}_{i}=\sigma(a) r+\mathbf{m}_{i}$. Note that we have $r+\mathbf{m}_{i}=\sigma(s)+\mathbf{m}_{i}$ and hence

$$
\sigma(a) r+\mathbf{m}_{i}=\sigma(a) \sigma(s)+\mathbf{m}_{i}=\sigma(a s)+\mathbf{m}_{i}
$$

since $\sigma$ is a homomorphism. By the previous paragraph, we thus have $s^{\prime}+\mathbf{m}_{i-1}=a s+\mathbf{m}_{i-1}$ and the proof is complete.

The module $N(\underline{\mathbf{m}})$ will be referred to as a double infinite string module. These modules should be compared with modules in [DGO, Section 3] and [LZ, Lemma 7].

Lemma 11. For $\underline{\mathbf{m}}, \underline{\mathbf{n}} \in{ }_{\infty} Q_{\infty}$ we have $N(\underline{\mathbf{m}}) \cong N(\underline{\mathbf{n}})$ if and only if there is $k \in \mathbb{Z}$ such that $\mathbf{m}_{i}=\mathbf{n}_{i+k}$ for all $i \in \mathbb{Z}$.

Proof. The "if" part is clear. To prove the "only if" part we consider an isomorphism $\varphi: N(\underline{\mathbf{m}}) \rightarrow N(\underline{\mathbf{n}})$ and let $v \in \mathbb{k}_{\mathbf{m}_{0}}$ be a nonzero element. We have $\varphi(v)=\sum_{i \in \mathbb{Z}} w_{i}$ with $w_{i} \in \mathbb{k}_{\mathbf{n}_{i}}$. As $\varphi(v) \neq 0$ we may define $l:=\max \left\{i \in \mathbb{Z}: w_{i} \neq 0\right\}$. As $\varphi$ is an isomorphism, we have $\mathbf{n}_{l}=\mathbf{m}_{0}$. Now the fact that $\mathbf{n}_{l-i}=\mathbf{m}_{-i}$ for all $i \in \mathbb{N}$ follows from Lemma 9. The fact that $\mathbf{n}_{l+i}=\mathbf{m}_{+i}$ for all $i \in \mathbb{N}$ follows by combining that $\varphi$ is a isomorphism and that the action of $Y$ is bijective on both $N(\underline{\mathbf{m}})$ and $N(\underline{\mathbf{n}})$.

Denote by $Q_{\infty}$ the set of all maps $\mathbb{Z}_{+}:=\{0,1,2, \ldots\} \rightarrow \max (R)$, $i \mapsto \mathbf{m}_{i}$, such that $\sigma\left(\mathbf{m}_{i}\right) \subset \mathbf{m}_{i+1}$ for all $i \in \mathbb{Z}_{+}$and, additionally, $\sigma(t) \in \mathbf{m}_{0}$. We will write $\underline{\mathbf{m}}:=\left(\mathbf{m}_{i}\right) \in Q_{\infty}$ if the collection $\left(\mathbf{m}_{i}\right)$ comes from such a map. 
For $\underline{\mathbf{m}} \in Q_{\infty}$ set

$$
N^{\uparrow}(\underline{\mathbf{m}}):=\bigoplus_{i \in \mathbb{Z}_{+}} \mathbb{k}_{\mathbf{m}_{i}}
$$

Then $N^{\uparrow}(\underline{\mathbf{m}})$ carries the natural structure of an $R$-module. For $i \in \mathbb{Z}_{+}$ define $X: \mathbb{k}_{\mathbf{m}_{i}} \rightarrow \mathbb{k}_{\mathbf{m}_{i+1}}$ as mapping $r+\mathbf{m}_{i}$ to $\sigma(t r)+\mathbf{m}_{i+1}$. Finally, set $Y \mathbb{k}_{\mathbf{m}_{0}}=0$ and for $i \in\{1,2,3, \ldots\}$ define $Y: \mathbb{k}_{\mathbf{m}_{i}} \rightarrow \mathbb{k}_{\mathbf{m}_{i-1}}$ as mapping $r+\mathbf{m}_{i}$ to $s+\mathbf{m}_{i-1}$ for some $s \in R$ such that $\sigma(s) \in r+\mathbf{m}_{i}$. Such an $s \in R$ exists because $\sigma$ is essential.

Lemma 12. The above defines on $N^{\uparrow}(\underline{\mathbf{m}})$ the structure of a weight A-module.

Proof. Mutatis mutandis the proof of Lemma 10.

The module $N^{\uparrow}(\underline{\mathbf{m}})$ will be referred to as a right infinite string module. Note that some right infinite string modules are subquotients of double infinite string modules. The weight $\mathbf{m}_{0}$ will be called the lowest weight.

Lemma 13. For $\underline{\mathbf{m}}, \underline{\mathbf{n}} \in Q_{\infty}$ we have $N^{\uparrow}(\underline{\mathbf{m}}) \cong N^{\uparrow}(\underline{\mathbf{n}})$ if and only if $\underline{\mathbf{m}}=\underline{\mathbf{n}}$.

Proof. Mutatis mutandis the proof of Lemma 11.

Denote by ${ }_{\infty} Q$ the set of all maps $\mathbb{Z}_{-}:=\{0,-1,-2, \ldots\} \rightarrow \max (R)$, $i \mapsto \mathbf{m}_{i}$, such that $\sigma\left(\mathbf{m}_{i-1}\right) \subset \mathbf{m}_{i}$ for all $i \in \mathbb{Z}_{-}$and, additionally, $t \in \mathbf{m}_{0}$. We will write $\underline{\mathbf{m}}:=\left(\mathbf{m}_{i}\right) \in{ }_{\infty} Q$ if the collection $\left(\mathbf{m}_{i}\right)$ comes from such a map.

For $\underline{\mathbf{m}} \in{ }_{\infty} Q$ set

$$
N^{\downarrow}(\underline{\mathbf{m}}):=\bigoplus_{i \in \mathbb{Z}_{-}} \mathbb{k}_{\mathbf{m}_{i}}
$$

Then $N^{\downarrow}(\underline{\mathbf{m}})$ carries the natural structure of an $R$-module. Set $X \mathbb{k}_{\mathbf{m}_{0}}=$ 0 and for $i \in\{-1,-2,-3, \ldots\}$ define $X: \mathbb{k}_{\mathbf{m}_{i}} \rightarrow \mathbb{k}_{\mathbf{m}_{i+1}}$ as mapping $r+\mathbf{m}_{i}$ to $\sigma(t r)+\mathbf{m}_{i+1}$. Finally, for $i \in \mathbb{Z}_{-}$define $Y: \mathbb{k}_{\mathbf{m}_{i}} \rightarrow \mathbb{k}_{\mathbf{m}_{i-1}}$ as mapping $r+\mathbf{m}_{i}$ to $s+\mathbf{m}_{i-1}$ for some $s \in R$ such that $\sigma(s) \in r+\mathbf{m}_{i}$. Such an $s \in R$ exists because $\sigma$ is essential.

Lemma 14. The above defines on $N^{\downarrow}(\underline{\mathbf{m}})$ the structure of a weight A-module.

Proof. Mutatis mutandis the proof of Lemma 10.

The module $N^{\downarrow}(\underline{\mathbf{m}})$ will be referred to as a left infinite string module. Note that some left infinite string modules are subquotients of double infinite string modules. The weight $\mathbf{m}_{0}$ will be called the highest weight. 
Lemma 15. For $\underline{\mathbf{m}}, \underline{\mathbf{n}} \in{ }_{\infty} Q$ we have $N^{\downarrow}(\underline{\mathbf{m}}) \cong N^{\downarrow}(\underline{\mathbf{n}})$ if and only if $\underline{\mathbf{m}}=\underline{\mathbf{n}}$.

Proof. Mutatis mutandis the proof of Lemma 11,

For $n \in \mathbb{Z}_{+}$denote by $Q_{n}$ the set of all maps $\{0,1, \ldots, n\} \rightarrow \max (R)$, $i \mapsto \mathbf{m}_{i}$, such that $\sigma\left(\mathbf{m}_{i}\right) \subset \mathbf{m}_{i+1}$ for all $i=0,1, \ldots, n-1$ and, additionally, $\sigma(t) \in \mathbf{m}_{0}$ and $t \in \mathbf{m}_{n}$. We will write $\underline{\mathbf{m}}:=\left(\mathbf{m}_{i}\right) \in Q_{n}$ if the collection $\left(\mathbf{m}_{i}\right)$ comes from such a map.

For $\underline{\mathbf{m}} \in Q_{n}$ set

$$
N_{n}(\underline{\mathbf{m}}):=\bigoplus_{i=0}^{n} \mathbb{k}_{\mathbf{m}_{i}} .
$$

Then $N_{n}(\underline{\mathbf{m}})$ carries the natural structure of an $R$-module. Set $X \mathbb{k}_{\mathbf{m}_{n}}=$ 0 and for $i \in\{0,1, \ldots, n-1\}$ define $X: \mathbb{k}_{\mathbf{m}_{i}} \rightarrow \mathbb{k}_{\mathbf{m}_{i+1}}$ as mapping $r+\mathbf{m}_{i}$ to $\sigma(t r)+\mathbf{m}_{i+1}$. Finally, set $Y \mathbb{k}_{\mathbf{m}_{0}}=0$ and for $i \in\{1,2, \ldots, n\}$ define $Y: \mathbb{k}_{\mathbf{m}_{i}} \rightarrow \mathbb{k}_{\mathbf{m}_{i-1}}$ as mapping $r+\mathbf{m}_{i}$ to $s+\mathbf{m}_{i-1}$ for some $s \in R$ such that $\sigma(s) \in r+\mathbf{m}_{i}$. Such an $s \in R$ exists because $\sigma$ is essential.

Lemma 16. The above defines on $N_{n}(\underline{\mathbf{m}})$ the structure of a weight A-module.

Proof. Mutatis mutandis the proof of Lemma 10 ,

The module $N_{n}(\underline{\mathbf{m}})$ will be referred to as a bounded string module. Note that some bounded string modules are subquotients of infinite string modules. The weight $\mathbf{m}_{0}$ will be called the lowest weight. The weight $\mathbf{m}_{n}$ will be called the highest weight.

Lemma 17. For $\underline{\mathbf{m}}, \underline{\mathbf{n}} \in Q_{n}$ we have $N_{n}(\underline{\mathbf{m}}) \cong N_{n}(\underline{\mathbf{n}})$ if and only if $\underline{\mathbf{m}}=\underline{\mathbf{n}}$.

Proof. Mutatis mutandis the proof of Lemma 11,

2.5. Simplicity of string modules. As we will see in this subsection, generically the string modules defined above are simple (this should be compared to [DGO, Section 3] and [LZ, Section 3]).

We call $\underline{\mathbf{m}}=\left(\mathbf{m}_{i}\right) \in{ }_{\infty} Q_{\infty}$ periodic provided that there is a nonzero $k \in \mathbb{Z}$ such that $\mathbf{m}_{i}=\mathbf{m}_{i+k}$ for all $i \in \mathbb{Z}$ and aperiodic otherwise.

Lemma 18. Let $\underline{\mathbf{m}} \in{ }_{\infty} Q_{\infty}$. Assume that there exist $m, n \in \mathbb{Z}$ such that $m<n$ and $\mathbf{m}_{m}=\mathbf{m}_{n}$. Then $\mathbf{m}_{m+i}=\mathbf{m}_{n+i}$ for all $i \in \mathbb{Z}_{-}$.

Proof. This follows directly from Lemma 5 ,

Proposition 19. For $\underline{\mathbf{m}} \in{ }_{\infty} Q_{\infty}$ the module $N(\underline{\mathbf{m}})$ is simple if and only if $\underline{\mathbf{m}}$ is aperiodic and $t \notin \mathbf{m}_{i}$ for all $i \in \mathbb{Z}$. 
Proof. We start with the "if" statement. If $\underline{\mathbf{m}}$ is aperiodic, then from Lemma 18 it follows that $\mathbf{m}_{i} \neq \mathbf{m}_{j}$ for all $i, j \gg 0$. Let $v \in N(\underline{\mathbf{m}})$ be a nonzero weight vector. If $t \notin \mathbf{m}_{i}$ for all $i \in \mathbb{Z}$, then the action of $X$ on $N(\underline{\mathbf{m}})$ is injective by Lemma 1 . For $k \gg 0$ weight components of $X^{k} v$ thus are of the form $r+\mathbf{m}_{i}$ for some $i \gg 0$. In particular, they all are different. Hence the submodule of $N(\underline{\mathbf{m}})$ generated by $v$ contains $N(\underline{\mathbf{m}})_{\mathbf{m}_{i}}$ for some $i \gg 0$. Note further that $t \notin \mathbf{m}_{i}$ for all $i \in \mathbb{Z}$ implies $\sigma(t) \notin \mathbf{m}_{i}$ for all $i \in \mathbb{Z}$. Hence the action of $Y$ on $N(\underline{\mathbf{m}})$ is injective as well by Lemma 1. The elements $X^{k}\left(r+\mathbf{m}_{i}\right)$ and $Y^{k}\left(r+\mathbf{m}_{i}\right), k \in \mathbb{Z}_{+}$, generate $N(\underline{\mathbf{m}})$. Therefore the submodule of $N(\underline{\mathbf{m}})$ generated by $v$ is the whole $N(\underline{\mathbf{m}})$ which proves that $N(\underline{\mathbf{m}})$ is simple.

Now we prove the "only if" statement. If $t \in \mathbf{m}_{i}$ for some $i$, then

$$
\bigoplus \mathbb{k}_{j \leqslant i} \mathbf{m}_{i}
$$

is a proper submodule of $N(\underline{\mathbf{m}})$ and hence $N(\underline{\mathbf{m}})$ is not simple.

If $\underline{\mathbf{m}}$ is periodic, say with some period $0 \neq k \in \mathbb{Z}$, then it is easy to check that $\left(1+\mathbf{m}_{0}\right)+\left(1+\mathbf{m}_{k}\right)$ generates a proper submodule in $N(\underline{\mathbf{m}})$ and hence $N(\underline{\mathbf{m}})$ is not simple.

Proposition 20. (i) For $\underline{\mathbf{m}} \in Q_{\infty}$ the module $N^{\uparrow}(\underline{\mathbf{m}})$ is simple if and only if $t \notin \mathbf{m}_{i}$ for all $i \in \mathbb{Z}_{+}$.

(ii) For $\underline{\mathbf{m}} \in{ }_{\infty} Q$ the module $N^{\downarrow}(\underline{\mathbf{m}})$ is simple if and only if $t \notin \mathbf{m}_{i}$ for all $i<0$.

(iii) For $\underline{\mathbf{m}} \in Q_{n}$ the module $N_{n}(\underline{\mathbf{m}})$ is simple if and only if $t \notin \mathbf{m}_{i}$ for all $i=0,1,2, \ldots, n-1$.

Proof. Mutatis mutandis the proof of Proposition 19.

\section{Category of Weight modules}

3.1. "Orbits" of $\sigma$. For $\mathbf{n}, \mathbf{m} \in \operatorname{Max}(R)$ write $\mathbf{n} \leftarrow \mathbf{m}$ provided that $\sigma(\mathbf{n}) \subset \mathbf{m}$. Let $\sim$ denote the minimal equivalence relation on $\operatorname{Max}(R)$ which contains the relation $\leftarrow$.

Because of Lemma 5 the relation $\leftarrow$ can be interpreted as a partially defined endomorphism (operating from the right to the left) of the set $\operatorname{Max}(R)$. With this interpretation, equivalence classes of $\sim$ can be though of as some kind of generalized "orbits" of the map $\leftarrow$. If $\sigma$ is an automorphism, then equivalence classes of $\sim$ are genuine orbits of the cyclic group generated by $\sigma$ acting on $\operatorname{Max}(R)$.

For $\mathbf{n}, \mathbf{m} \in \operatorname{Max}(R)$ write $\mathbf{n} \stackrel{0}{\leftarrow} \mathbf{m}$ provided that $\mathbf{n}=\mathbf{m}$. For $k \in \mathbb{N}$ write $\mathbf{n} \stackrel{k}{\leftarrow} \mathbf{m}$ provided that there exist $\mathbf{m}_{i} \in \operatorname{Max}(R), i=1,2, \ldots, k-1$, 
such that

$$
\mathbf{n} \leftarrow \mathbf{m}_{1} \leftarrow \mathbf{m}_{2} \leftarrow \cdots \leftarrow \mathbf{m}_{k-1} \leftarrow \mathbf{m}
$$

In particular, $\mathbf{n} \leftarrow \mathbf{m}$ if and only if $\mathbf{n} \leftarrow \mathbf{m}$. Further, we have $\mathbf{n} \sim \mathbf{m}$ if and only if there is $\mathbf{k} \in \operatorname{Max}(R)$ and $m, n \in \mathbb{Z}_{+}$such that $\mathbf{k} \stackrel{m}{\leftarrow} \mathbf{m}$ and $\mathbf{k} \stackrel{n}{\leftarrow} \mathbf{n}$. The equivalence classes $S \in \operatorname{Max}(R) / \sim$ fall into two different cases.

Rooted classes, that is classes $S$ for which there exists $\mathbf{n} \in S$ such that $\sigma(\mathbf{k}) \not \subset \mathbf{n}$ for all $\mathbf{k} \in \operatorname{Max}(R)$. In this case $S$ consists of all $\mathbf{m} \in \operatorname{Max}(R)$ such that $\mathbf{n} \stackrel{k}{\leftarrow} \mathbf{m}$ for some $k \in \mathbb{Z}_{+}$.

Unrooted classes, that is classes $S$ for which $\mathbf{n}$ as in the previous paragraph does not exist. In this case for any $\mathbf{n} \in S$ the class $S$ consists of all $\mathbf{m} \in \operatorname{Max}(R)$ for which there exist $\mathbf{k} \in S$ and $k, l \in \mathbb{Z}_{+}$ such that $\mathbf{k} \stackrel{k}{\leftarrow} \mathbf{n}$ and $\mathbf{k} \stackrel{l}{\leftarrow} \mathbf{m}$.

From this point of view each $S \in \operatorname{Max}(R) / \sim$ is a lower semilattice.

3.2. Standard decomposition of the category of weight modules. Let $\mathfrak{W}$ denote the full subcategory of $A$-mod consisting of all weight modules. For $S \in \operatorname{Max}(R) / \sim$ denote by $\mathfrak{W}_{S}$ the full subcategory of $\mathfrak{W}$ consisting of all modules $M$ such that $\operatorname{supp}(M) \subset S$. Then we have the following standard fact (cf. [DGO, Proposition 1.5]):

Lemma 21. Assume $\sigma$ is essential. Then we have the decomposition

$$
\mathfrak{W} \cong \bigoplus_{S \in \operatorname{Max}(R) / \sim} \mathfrak{W}_{S}
$$

Proof. This follows directly from Lemmata 2 and 9 .

As we will see, one major difference between GWAs and wGWAs is that for the former algebras the categories $\mathfrak{W}_{S}$ are usually indecomposable, while for the latter algebras the categories $\mathfrak{W}_{S}$ are usually decomposable.

\section{Simple Weight modules}

\subsection{Support of a simple weight module.}

Proposition 22. Let $M$ be a simple weight module. Then there is

$$
\underline{\mathbf{m}} \in{ }_{\infty} Q_{\infty} \bigcup Q_{\infty} \bigcup{ }_{\infty} Q \bigcup \bigcup_{n \in \mathbb{Z}^{+}} Q_{n}
$$

such that $\operatorname{supp}(M) \subset \underline{\mathbf{m}}$. 
Proof. We start with the easy observation that

$$
A=\sum_{i, j \in \mathbb{Z}_{+}} Y^{i} R X^{j}
$$

which follows directly from (1.1) (note that the sum in (4.1) is not claimed to be direct). Now observe the following:

Lemma 23. Let $M$ be a simple weight module. Then for any different $\mathbf{m}, \mathbf{n} \in \operatorname{supp}(M)$ there exists $k \in \mathbb{Z}_{+}$and $\mathbf{k}_{1}, \mathbf{k}_{2}, \ldots, \mathbf{k}_{k} \in \operatorname{supp}(M)$ such that either we have $\mathbf{m} \leftarrow \mathbf{k}_{1} \leftarrow \cdots \leftarrow \mathbf{k}_{k} \leftarrow \mathbf{n}$ or we have $\mathbf{n} \leftarrow \mathbf{k}_{1} \leftarrow \cdots \leftarrow \mathbf{k}_{k} \leftarrow \mathbf{m}$

Proof. This follows immediately combining (4.1), Lemma 2, Lemma 9 and the property $A M_{\mathbf{m}}=M$.

From (4.1) it follows that $\operatorname{supp}(M)$ is at most countable, say $\operatorname{supp}(M)=$ $\left\{\mathbf{n}_{1}, \mathbf{n}_{2}, \ldots\right\}$. Set $\mathbf{m}_{1}:=\mathbf{n}_{1}$ and define $K_{1}$ as the maximal sequence $\left(\ldots, \mathbf{m}_{-1}, \mathbf{m}_{0}, \mathbf{m}_{1}\right)$, finite or infinite, such that all $\mathbf{m}_{i} \in \operatorname{supp}(M)$ and $\mathbf{m}_{i} \leftarrow \mathbf{m}_{i+1}$ for all $i \leqslant 0$ appearing in the sequence. Define now $K_{s}$ for $s>1$ inductively as follows: If all elements of $\operatorname{supp}(M)$ appear already in $K_{s-1}$, set $K_{s}:=K_{s-1}$. Otherwise, choose $i$ minimal such that $\mathbf{n}_{i} \in \operatorname{supp}(M)$ does not appear in $K_{s-1}$. Let $\mathbf{m}$ be the rightmost element of $K_{s-1}$. Then from Lemma 23 it follows that

$$
\mathbf{m} \leftarrow \mathbf{k}_{1} \leftarrow \cdots \leftarrow \mathbf{k}_{k} \leftarrow \mathbf{n}_{i}
$$

for some $k \in \mathbb{Z}_{+}$and $\mathbf{k}_{1}, \mathbf{k}_{2}, \ldots, \mathbf{k}_{k} \in \operatorname{supp}(M)$ and we can set

$$
K_{s}:=\left(K_{s-1}, \mathbf{k}_{1}, \ldots, \mathbf{k}_{k}, \mathbf{n}_{i}\right) .
$$

Then $\operatorname{supp}(M)$ is, by construction, the limit of $K_{i}$ when $i \rightarrow \infty$, and it obviously has the required form.

4.2. Band modules. Let $\underline{\mathbf{m}}=\left\{\mathbf{m}_{i}\right\}$ be doubly infinite and periodic with the minimal period $k \in\{1,2,3, \ldots\}$. Set $\mathbf{m}:=\mathbf{m}_{0}$ and $\mathbb{k}:=\mathbb{k}_{\mathbf{m}}$. Note that $\sigma$, being essential, induces for each $i>0$ an isomorphism $\mathbb{k} \cong \mathbb{k}_{\mathbf{m}_{i}}$ by sending $r+\mathbf{m}$ to $\sigma^{i}(r)+\mathbf{m}_{i}$. Denote the latter isomorphism by $\tau_{i}$ and also denote by $\bar{\sigma}$ the automorphism of $\mathbb{k}$ induced by $\tau_{k}$. Set $\tau_{0}:=\mathrm{id}_{\mathbb{k}}$.

Consider the skew Laurent polynomial ring $\mathbf{P}:=\mathbb{k}\left[\alpha, \alpha^{-1}, \bar{\sigma}\right]$ given by $\alpha(r+\mathbf{m})=\bar{\sigma}(r+\mathbf{m}) \alpha$ for $r \in R$. This ring is a principal ideal domain (both left and right). For an irreducible element $f \in \mathbf{P}$ denote by $L_{f}:=\mathbf{P} / \mathbf{P} f$ the corresponding simple module. Note that $L_{f} \cong L_{\tilde{f}}$ if and only if $f$ and $\tilde{f}$ are similar in $\mathbf{P}$. Note also that $L_{f}$ is finite dimensional over $\mathbb{k}$.

Define the weight $A$-module $M^{f}$ with

$$
\operatorname{supp}\left(M^{f}\right):=\left\{\mathbf{m}_{-(k-1)}, \ldots \mathbf{m}_{-2}, \mathbf{m}_{-1}, \mathbf{m}_{0}\right\}
$$


as follows: for $i \in\{0,1, \ldots, k-1\}$ define the $R$-module $M_{\mathbf{m}_{-i}}^{f}$ as ${ }^{\tau_{i}} L_{f}$, that is $M_{\mathbf{m}_{-i}}^{f}=L_{f}$ as the vector space but the action of $R$ is twisted by $\tau_{i}$, that is $\left(r+\mathbf{m}_{-i}\right) \cdot v=\tau_{i}(r+\mathbf{m}) v$ for $r \in R$ and $v \in L_{f}$. For $i \in\{0,1, \ldots, k-2\}$ define the action of $Y$ from $L_{f}=M_{\mathbf{m}_{-i}}^{f}$ to $L_{f}=M_{\mathbf{m}_{-i-1}}^{f}$ as the identity. Define the action of $Y$ from $M_{\mathbf{m}_{-(k-1)}}^{f}$ to $M_{\mathbf{m}_{0}}^{f}$ as the action of $\alpha^{-1}$. In particular, the action of $Y$ on $M_{\mathbf{m}_{-i}}^{f}$ is bijective. Define the action of $X$ on $Y v$ as $\sigma(t) v$.

Lemma 24. The above defines on $M^{f}$ the structure of a simple weight $A$-module, moreover, $M^{f} \cong M^{\tilde{f}}$ if and only if $f$ and $\tilde{f}$ are similar.

Proof. To prove that $M^{f}$ is an $A$-module, we have to check the defining relations. They all are immediate from the definitions. To prove simplicity let $v \in M^{f}$ be a nonzero weight element. As the action of $Y$ is bijective, we may assume $v \in M_{\mathbf{m}}^{f}$. The linear operator $Y^{k}$ on $M_{\mathbf{m}}^{f}$ is bijective by definition and hence defines on $M_{\mathbf{m}}^{f}$ the structure of a $\mathbf{P}$-module which is isomorphic to $L_{f}$ by construction. Therefore the $A$-submodule generated by $v$ contains $M_{\mathbf{m}}^{f}$ and thus the whole of $M^{f}$ as the action of $Y$ is bijective. The claim about isomorphism is clear.

Define the weight $A$-module $N^{f}$ with

$$
\operatorname{supp}\left(N^{f}\right):=\left\{\mathbf{m}_{0}, \mathbf{m}_{1}, \mathbf{m}_{2}, \ldots, \mathbf{m}_{k-1}\right\}
$$

as follows: for $i \in\{0,1, \ldots, k-1\}$ define the $R$-module $N_{\mathbf{m}_{i}}^{f}$ as ${ }^{\tau_{i}^{-1}} L_{f}$, that is $M_{\mathbf{m}_{i}}^{f}=L_{f}$ as the vector space but the action of $R$ is twisted by $\tau_{i}^{-1}$, that is $\tau_{i}(r+\mathbf{m}) \cdot v=(r+\mathbf{m}) v$ for $r \in R$ and $v \in L_{f}$. For $i=0,1,2, \ldots, k-2$ define the action of $X$ from $M_{\mathbf{m}_{i}}^{f}=L_{f}$ to $M_{\mathbf{m}_{i+1}}^{f}=L_{f}$ as the identity. Define the action of $X$ from $M_{\mathbf{m}_{k-1}}^{f}$ to $M_{\mathbf{m}_{0}}^{f}$ as the action of $\alpha^{-1}$. Then the action of $X$ is bijective. Define the action of $Y$ on $X v$ as $t v$.

Lemma 25. The above defines on $N^{f}$ the structure of a simple weight $A$-module, moreover, $N^{f} \cong N^{\tilde{f}}$ if and only if $f$ and $\tilde{f}$ are similar.

Proof. Mutatis mutandis the proof of Lemma 24.

Lemma 26. We have $M^{f} \cong N^{\tilde{f}}$ if and only if $f$ is similar to $\tilde{f}$ and for every $i=0,1,2, \ldots, k-1$ we have $t \notin \mathbf{m}_{i}$.

Proof. If $M^{f} \cong N^{\tilde{f}}$ the both $X$ and $Y$ act bijectively on $M^{f}$ and hence for every $i=0,1,2, \ldots, k-1$ we have $t \notin \mathbf{m}_{i}$. Further, we have $M_{\mathbf{m}}^{f} \cong N_{\mathbf{m}}^{\tilde{f}}$ as modules over the subalgebra generated by $R$ and $X^{k}$. It is easy to check that the latter action defines on $M_{\mathbf{m}}^{f}$ and $N_{\mathbf{m}}^{\tilde{f}}$ the 
structures of $\mathbf{P}$-modules (given by $f$ and $\tilde{f}$, respectively) which thus must be isomorphic. This yields that $f$ and $\tilde{f}$ are similar.

Assume now that $f$ is similar to $\tilde{f}$ and for every $i=0,1,2, \ldots, k-1$ we have $t \notin \mathbf{m}_{i}$. Then the actions of both $X$ and $Y$ on both $M^{f}$ and $N^{\tilde{f}}$ are bijective. As the action of $X^{k} Y^{k}$ on both $M_{\mathbf{m}}^{f}$ and $N_{\mathbf{m}}^{\tilde{f}}$ is given by some element of $r$ and is non-zero, $X^{k}$ is an inverse of $Y^{k}$ up to a scalar. The claim $M^{f} \cong N^{\tilde{f}}$ now follows by comparing the definitions of $M^{f}$ and $N^{\tilde{f}}$.

4.3. Classification of simple weight modules. Our main result is the following theorem

Theorem 27. Each simple weight A-module is either a string module or a band module.

To prove this theorem we will need several lemmata.

Lemma 28. The action of $X$ on a simple weight $A$-module is either injective or locally nilpotent.

Proof. Assume that $V$ is a simple weight $A$-module and $v \in V$ is nonzero and such that $X v=0$. Write $v=\sum_{\mathbf{m}} v_{\mathbf{m}}$, where $v_{\mathbf{m}} \in V_{\mathbf{m}}$. From Lemmata 2 and 5 it follows that for $\mathbf{m} \neq \mathbf{n}$ the weights of the vectors $X v_{\mathbf{m}}$ and $X v_{\mathbf{n}}$ do not intersect. Hence $X v=0$ implies $X v_{\mathbf{m}}=0$ for all $\mathbf{m}$. Therefore we may assume that $v$ is a weight vector, say of weight $\mathbf{m}$.

From (4.1) it then follows that $V=A v=\sum_{i \in \mathbb{Z}_{+}} Y^{i} \mathbb{k}_{\mathbf{m}} v$. From (1.1) and $X v=0$ we obtain that $X^{i+1} Y^{i} \mathbb{k}_{\mathbf{m}} v=0$. The claim follows.

Lemma 29. The action of $Y$ on a simple weight A-module is either injective or locally nilpotent.

Proof. Let $V$ be a simple weight $A$-module. We have a rough description of $\operatorname{supp}(M)$ given in Proposition 22, According to this description, we have one of the following two cases.

Case 1. Assume that for any $\mathbf{m} \in \operatorname{supp}(M)$ there exists at most one $\mathbf{n} \in \operatorname{supp}(M)$ such that $\sigma(\mathbf{m}) \subset \mathbf{n}$. Assume that $v \in V$ is nonzero and such that $Y v=0$. Similarly to the proof of Lemma 28, we may assume that $v$ is weight. We claim that $V=\sum_{i \in \mathbb{Z}_{+}} X^{i} \mathbb{k}_{\mathbf{m}} v$. Denote the right hand side by $N$, it is clearly nonzero and stable under the action of $X$. From our assumption for Case 1 we also have that all $X^{i} v$ are weight vectors. As $\sigma$ is essential, using (1.1) it follows that $R X^{i} v=X^{i} \mathbb{k}_{\mathbf{m}} v$ and hence $N$ is stable under the action of $R$. The latter and $Y v=0$ implies that $N$ is a non-trivial $A$-submodule of $V$ and hence coincides 
with $V$ as $V$ is simple. It is easy to check that $Y$ acts locally nilpotently on $N$.

Case 2. There is a unique $\mathbf{m} \in \operatorname{supp}(M)$ for which there is more than one $\mathbf{n} \in \operatorname{supp}(M)$ such that $\sigma(\mathbf{m}) \subset \mathbf{n}$, more precisely, there are exactly two different $\mathbf{n}_{1}, \mathbf{n}_{2} \in \operatorname{supp}(M)$ such that $\sigma(\mathbf{m}) \subset \mathbf{n}_{1}$ and $\sigma(\mathbf{m}) \subset \mathbf{n}_{2}$. Assume that $v \in V$ is nonzero and such that $Y v=0$. We want to show that we may assume that $v$ is weight. The only case when for this statement the argument used before does not work is when $v=v_{1}+v_{2}$ with $v_{i} \in V_{\mathbf{n}_{i}}, i=1,2$. We nevertheless claim that $Y v_{i}=0$ for $i=1,2$. Indeed, if not, then we have $Y v_{1}=-Y v_{2}$. As $\sigma$ is essential, we can rewrite this as $Y v_{1}=Y r v_{2}$ for some $r \in R$. Applying $X$ we get $X Y v_{1}=X Y r v_{2}$. We have $X Y v_{1} \in V_{\mathbf{n}_{1}}$ and $X Y r v_{2} \in V_{\mathbf{n}_{2}}$ which implies $X Y v_{1}=X Y r v_{2}=0$ since $\mathbf{n}_{1} \neq \mathbf{n}_{2}$. If $Y v_{1} \neq 0$, then from Lemma 28 we have that $X$ acts locally nilpotently on $V$ and $V=\sum_{i \in \mathbb{Z}_{+}} Y^{i} \mathbb{k}_{\mathbf{m}} Y v_{1}$. Combining Proposition 22 with Lemma 9 it thus follows that $V$ falls into Case 1, a contradiction. Therefore we may assume that $v$ is a weight vector.

From Lemma 1 it thus follows that there exists $\mathbf{k} \in \operatorname{supp}(M)$ such that $\sigma(t) \in \mathbf{k}$. If $Y M_{\mathbf{k}}=0$, the action of $Y$ on $M$ is clearly locally nilpotent. If $Y M_{\mathbf{k}} \neq 0$, then $Y M_{\mathbf{k}} \subset M_{\mathbf{l}}$ for some $\mathbf{l} \in \operatorname{supp}(M)$ and there is a nonzero $w \in M_{1}$ such that $X v=0$. From the proof of Lemma 28 it follows that in this case $M=\sum_{i \geqslant 0} Y^{i} R M_{\mathbf{l}}$. As one of the weights $\mathbf{n}_{1}$ or $\mathbf{n}_{2}$ cannot belong to the support of $\sum_{i \geqslant 0} Y^{i} R M_{\mathbf{l}}$, this case does not occur. The claim of the lemma follows.

Proof of Theorem 27. Let $V$ be a simple weight $A$-module. We prove the result using a case-by-case analysis.

Case 1. Assume that the action of both $X$ and $Y$ on $V$ is locally nilpotent. Let further $v \in V$ be a weight element such that $X v=0$. Then from the proof of Lemma 28 we have that $V=\sum_{i=0}^{k} Y^{i} R v$ for some minimal $k$ and it is straightforward to check that $V$ is a bounded string module.

Case 2. Assume that the action of $X$ on $V$ is locally nilpotent while the action of $Y$ on $V$ is injective. Let $v \in V$ be a weight element, say of weight $\mathbf{m}$, such that $X v=0$.

Subcase 2a. Assume further that for each $i>0$ the weight of the weight element $Y^{i} v$ is different from $\mathbf{m}$. Let $I$ be the left ideal of $A$ generated by $\mathbf{m}$ and $X$. Then $A / I$ surjects onto $V$ via the unique map sending $1+I$ to $v$. At the same time, using the action of $A$ on $A / I$ in the basis $\left\{Y^{i} v: i \in \mathbb{Z}_{+}\right\}$it is clear that $A / I$ is a right bounded string module. Moreover, $A / I$ is simple for otherwise it would have a string 
submodule and the quotient (which would also surject onto $V$ ) would be a bounded string module.

Subcase 2b. Assume that there is a minimal $k>0$ such that the weight of the weight element $Y^{k} v$ equals $\mathbf{m}$. Let $I$ be the left ideal of $A$ generated by $\mathbf{m}$ and $X$. Then $A / I$ surjects onto $V$ via the unique map sending $1+I$ to $v$. Using the action of $A$ on $A / I$ in the basis $\left\{Y^{i} v: i \in \mathbb{Z}_{+}\right\}$it is clear that $A / I$ is a right bounded string module. However, this string module is not simple as $t \in \mathbf{m}$. Therefore $V$ is a quotient of the weight module $A / I$ modulo some proper weight submodule. This implies that for every $\mathbf{n} \in \operatorname{supp}(V)$ the space $V_{\mathbf{n}}$ is finite dimensional over $\mathbb{k}_{\mathbf{n}}$. As the action of $Y$ is injective, it follows that all weight spaces have the same dimension and thus the action of $Y$ is bijective. Now it clear that $V$ is a band module.

Case 3. Assume that the action of $Y$ on $V$ is locally nilpotent while the action of $X$ on $V$ is injective. Let $v \in V$ be a weight element, say of weight $\mathbf{m}$, such that $Y v=0$.

Subcase 3a. Assume that $\operatorname{supp}(V)$ is infinite. Then from Proposition 22 it follows that for each $i \in \mathbb{Z}_{+}$the element $X^{i} v$ is a weight element, say of weight $\mathbf{m}_{i}$, and, moreover, $\mathbf{m}_{i} \neq \mathbf{m}_{j}$ if $i \neq j$. The linear span of $R X^{i} v, i \in \mathbb{Z}_{+}$, is invariant with respect to the action of $Y$ and hence coincides with $V$. Therefore $V$ is a left bounded string module.

Subcase 3b. Assume that $\operatorname{supp}(V)$ is finite. Then, using the same arguments to the ones used for Subcase 2b, one shows that $V$ is a band module.

Case 4. Assume that the action of both $Y$ and $X$ on $V$ are injective.

Subcase 4a. Assume that $\operatorname{supp}(V)$ is infinite. Using Proposition 22 we may choose $\mathbf{m} \in \operatorname{supp}(V)$ such that for any $i \in \mathbb{N}$ the weight of the weight element $Y^{i} v$, where $v$ is a nonzero element in $V_{\mathbf{m}}$ is different from $\mathbf{m}$. Then, again from Proposition 22, it follows that $X^{i} v$ is a weight vector for each $i \in \mathbb{N}$. Using the fact that $\sigma$ is essential one now checks that the linear span of $v$ and $X^{i} v, Y^{i} v, i \in \mathbb{N}$, is a submodule and hence coincides with $V$. It follows that $V$ is a doubly infinite string module.

Subcase 4 b. Assume that $\operatorname{supp}(V)$ is finite. Then, using the same arguments to the ones used for Subcase 2b, one shows that $V$ is a band module. The claim of the theorem follows.

4.4. Weight modules that are of finite length over $R$. Let $V$ be a simple weight $A$-module. A natural analogue of finite dimensionality 
for $V$ is the requirement that $V$ is of finite length when viewed as an $R$-module. Directly from Theorem 27] we get:

Corollary 30. The only simple weight A-modules which are of finite length over $R$ are bounded string modules and band modules.

\section{Simple Finite Dimensional MODUleS}

5.1. Setup and definition. Let $A$ be a wGWA. In this section we assume that $R$ is an algebra over some field $\mathbb{k}$ and that $\sigma$ is an algebra endomorphism, in particular, that it is $\mathbb{k}$-linear. Then every $A$-module $M$ carries the natural structure of a vector space over $\mathbb{k}$ and we say that $M$ is finite dimensional if it is finite dimensional over $\mathbb{k}$.

From the definition of a finite dimensional $A$-module it is clear that each finite dimensional $A$-module is a generalized weight module and that the category of finite dimensional $A$-modules is Krull-Schmidt. In Theorem 31 below we will show that simple finite dimensional $A$ modules are, in fact, weight modules.

5.2. Simple finite dimensional modules. Our main result in this section is the following statement which reduces classification of simple finite dimensional $A$-module to Theorem 27.

Theorem 31. Assume that $R$ is an algebra over some field $\mathbb{k}$ and that $\sigma$ is essential and $\mathbb{k}$-linear. Then every simple finite dimensional $A$ module is a weight module.

5.3. Preliminary observations. As usual, we assume that $\sigma$ is essential. Let $M$ be a finite dimensional $A$-module. Then we have

$$
M=\bigoplus_{\mathbf{m} \in \operatorname{supp}(M)} M^{\mathbf{m}},
$$

$\operatorname{supp}(M)$ is finite and, moreover, each $M^{\mathbf{m}}$ is finite dimensional over $\mathbb{k}$, in particular, there is $k \in \mathbb{N}$ such that $M^{\mathbf{m}}$ is annihilated by $\mathbf{m}^{k}$. Further, using the fact that maximal ideals are prime, we obtain

$$
X M^{\mathbf{m}} \subset \bigoplus_{\mathbf{n}: \sigma(\mathbf{m}) \subset \mathbf{n}} M^{\mathbf{n}} \quad \text { and } \quad Y M^{\mathbf{m}} \subset \bigoplus_{\mathbf{n}: \sigma(\mathbf{n}) \subset \mathbf{m}} M^{\mathbf{n}}
$$

by exactly the same arguments as used in the proofs of Lemma 2 and Lemma 5, respectively. From Lemma 5 we know that the direct sum on the right in (5.1) has at most one summand. Similarly to Lemma 1 we have that $X v=0$ for some $v \in M^{\mathbf{m}}, v \neq 0$, implies $t \in \mathbf{m}$ and, analogously, $Y v=0$ implies $\sigma(t) \in \mathbf{m}$. 
Note that, if $\sigma(\mathbf{n}) \subset \mathbf{m}$, then $Y$ maps $M^{\mathbf{m}}$ to $M^{\mathbf{n}}$ by the above and, moreover, it maps $M_{\mathbf{m}}$ to $M_{\mathbf{n}}$ by the obvious computation using (1.1). In other words, the action of $Y$ preserves the weight part

$$
\mathrm{wt}(M):=\bigoplus_{\mathbf{m} \in \operatorname{supp}(M)} M_{\mathbf{m}}
$$

of $M$.

Let $\Gamma$ be the finite oriented graph whose vertices are elements of the finite set $\operatorname{supp}(M)$ and arrows $\mathbf{n} \leftarrow \mathbf{m}$ are defined as in Subsection 3.1, i.e. represent the inclusion $\sigma(\mathbf{n}) \subset \mathbf{m}$. By Lemma 10, each vertex of our graph is the source of at most one arrow, in particular, the number of arrows does not exceed the number of vertices.

5.4. Proof of Theorem 31. Consider first the case when $\Gamma$ contains some vertex $\mathbf{n}$ which is not the target of any arrow in $\Gamma$. Let $v \in M_{\mathbf{m}}$ be non-zero. Then $X v=0$ by (5.1) and hence (4.1) implies

$$
M=\sum_{i \in \mathbb{Z}_{+}} Y^{i} R v
$$

Now $R v \subset M_{\mathbf{m}}$ and since the action of $Y$ preserves wt $(M)$, we obtain that $M=\operatorname{wt}(M)$ is a weight module.

Assume now that each vertex in $\Gamma$ is the target of some arrow. Then the number of arrows and vertices in $\Gamma$ coincide and hence each vertex is the source of exactly one arrow and the target of exactly one arrow. From (5.1) and simplicity of $M$ it follows that $\Gamma$ is an oriented cycle, say

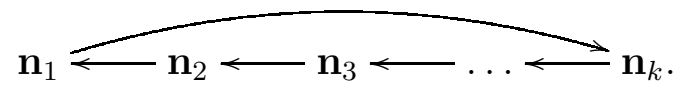

For $i=1,2, \ldots, k$ set $l_{i}:=\operatorname{dim}_{\mathbb{k}} M_{\mathbf{n}_{i}}$.

If some $M^{\mathbf{n}_{i}}$ contains a non-zero $v$ such that $X v=0$, then we have $X R v=\sigma(R) X v=0$ by (1.1). Therefore $M_{\mathbf{n}_{i}}$ contains a non-zero $w$ such that $X w=0$ and the same arguments as above show that $M$ is a weight module.

It remains to consider the case when $X$ acts injectively on $M$. Since $M$ is finite dimensional over $\mathbb{k}$ and $X$ is $\mathbb{k}$-linear (as $\sigma$ is $\mathbb{k}$-linear), we have that $X$ acts bijectively on $M$. Then any $v \in M_{\mathbf{n}_{i}}$ is of the form $v=X w$ for some $w \in M^{\mathbf{n}_{i-1}}$ (by convention, we have $\mathbf{n}_{0}=\mathbf{n}_{k}$ ). Using (1.1), we compute:

$$
X \mathbf{n}_{i-1} w=\sigma\left(\mathbf{n}_{i-1}\right) X w \subset \mathbf{n}_{i} v=0 .
$$

As $X$ acts injectively, we obtain $\mathbf{n}_{i-1} w=0$, which implies $w \in M_{\mathbf{n}_{i-1}}$. Therefore $l_{i-1} \geqslant l_{i}$ and the fact that the graph $\Gamma$ is a cycle implies $l_{1}=l_{2}=\cdots=l_{k}$. This means that the action of $X$ preserves $\operatorname{wt}(M)$. Since the action of $R$ obviously preserves wt $(M)$, it follows that wt $(M)$ 
is an $A$-submodule and thus coincides with $M$ by simplicity of the latter.

\section{Application to generalized Heisenberg Algebras}

6.1. General reduction. In this section we consider a number of concrete applications of the above results to classifications of simple weight modules over generalized Heisenberg algebra $\mathcal{H}(f)$ defined in the introduction, where $f(h) \in \mathbb{C}[h]$. Note that the element $z$ is central in $\mathcal{H}(f)$ and hence, by Schur's lemma, it acts as a scalar on each simple $\mathcal{H}(f)$-module.

Fix $\dot{z} \in \mathbb{C}$ and consider the quotient $\mathcal{H}(f)_{\dot{z}}$ of $\mathcal{H}(f)$ by the principal central ideal generated by $(z-\dot{z})$. Note that the algebra $\mathcal{H}(f)_{\dot{z}}$ is a wGWA for $R=\mathbb{C}[h], t=h+\dot{z}$ and $\sigma: R \rightarrow R$ defined by $\sigma(h)=f(h)$. Clearly, $\sigma$ is essential.

For $\chi \in \mathbb{C}$ we denote by $\mathbf{m}_{\chi}$ the maximal ideal $(h-\chi)$ in $R$ and in this way identify $\operatorname{Max}(R)$ with $\mathbb{C}$. For $\chi \in \mathbb{C}$ the ideal $\sigma\left(\mathbf{m}_{\chi}\right)$ is generated by $f(h)-\chi$. Therefore, for $\eta \in \mathbb{C}$ we have $\sigma\left(\mathbf{m}_{\chi}\right) \subset \mathbf{m}_{\eta}$ if and only if $f(\eta)=\chi$. Conversely, $\mathbf{m}_{f(\chi)}$ is the unique maximal ideal such that $\sigma\left(\mathbf{m}_{f(\chi)}\right) \subset \mathbf{m}_{\chi}$. Note that all this works even if $f$ is constant.

We have $t=h+\dot{z} \in \mathbf{m}_{-\dot{z}}$ and $t \notin \mathbf{m}_{\chi}$ for $\chi \neq-\dot{z}$. We also have $\sigma(t)=f(h)+\dot{z} \in \mathbf{m}_{\chi}$ if and only if $f(\chi)=-\dot{z}$.

Denote by $\Phi$ the oriented graph with vertices $\mathbb{C}$ and arrows $f(\chi) \leftarrow \chi$ for all $\chi \in \mathbb{C}$. Theorem 27 reduces classification of simple weight $\mathcal{H}(f)_{\dot{z}}$-modules to description of the dynamics of the action of the transformation $\chi \mapsto f(\chi)$ of $\mathbb{C}$, that is oriented paths in the graph $\Phi$.

6.2. Degree zero case. If $f$ has degree zero, then $f$ is a constant polynomial, say with value $\theta$. Assume first that $\theta \neq-\dot{z}$. Then from Theorem 27 we have that $\mathcal{H}(f)_{\dot{z}}$ has a unique infinite dimensional simple weight module, namely $N^{\downarrow}(\underline{\mathbf{m}})$, where

$$
\underline{\mathbf{m}}=\left(\ldots, \mathbf{m}_{\theta}, \mathbf{m}_{\theta}, \mathbf{m}_{\theta}, \mathbf{m}_{-\dot{z}}\right) \text {; }
$$

and a family of one-dimensional simple weight modules with support $\mathbf{m}_{\theta}$ indexed by $c \in \mathbb{C}^{*}$. Each module from the latter family coincides with $R /(h-\theta)$ as $R$-modules, on the module indexed by $c$ the element $X$ acts as $c$ and the element $Y$ acts as $\frac{\theta+\dot{z}}{c}$.

If $\theta=-\dot{z}$, then from Theorem 27 we have that $\mathcal{H}(f)_{\dot{z}}$ has the one dimensional simple weight module which coincides with $R /(h-\theta)$ as an $R$-module and on which both $X$ and $Y$ act as zero and, additionally, exactly two families of non-isomorphic simple weight modules indexed 
by $\mathbb{C}^{*}$, all modules having dimension one. All modules in both families coincide with $R /(h-\theta)$ as $R$-modules. On modules of the first family $X$ acts as $c \in \mathbb{C}^{*}$ and $Y$ as zero. On modules of the second family $Y$ acts as $c \in \mathbb{C}^{*}$ and $X$ as zero.

6.3. Degree one case. If $f$ has degree one and $f(h) \neq h$ (the latter means that $A$ is not commutative), then for every $\chi \in \mathbb{C}$ there is a unique $\eta \in \mathbb{C}$ such that $f(\eta)=\chi$. Let $\alpha \in \mathbb{C}$ be the unique number such that $f(\alpha)=-\dot{z}$. From Theorem 27 it thus follows that simple weight modules over $\mathcal{H}(f)_{\dot{z}}$ are: $N^{\downarrow}(\underline{\mathbf{m}})$ where

$$
\underline{\mathbf{m}}=\left(\ldots, \mathbf{m}_{f(f(f(-\dot{z})))}, \mathbf{m}_{f(f(-\dot{z}))}, \mathbf{m}_{f(-\dot{z})}, \mathbf{m}_{-\dot{z}}\right) ;
$$

$N^{\uparrow}(\underline{\mathbf{m}})$ where

$$
\underline{\mathbf{m}}=\left(\mathbf{m}_{\alpha}, \mathbf{m}_{\alpha_{1}}, \mathbf{m}_{\alpha_{2}}, \mathbf{m}_{\alpha_{3}}, \ldots\right)
$$

with $f\left(\alpha_{i}\right)=\alpha_{i-1}$ for $i=1,2, \ldots$; and $N(\underline{\mathbf{m}})$ where

$$
\underline{\mathbf{m}}=\left(\ldots, \mathbf{m}_{\beta_{-2}}, \mathbf{m}_{\beta_{-1}}, \mathbf{m}_{\beta_{0}}, \mathbf{m}_{\beta_{1}}, \mathbf{m}_{\beta_{2}}, \ldots\right)
$$

with $f\left(\beta_{i}\right)=\beta_{i-1}$ and $\beta_{i} \neq-\dot{z}$ for $i \in \mathbb{Z}$.

Note that in this case $\mathcal{H}(f)_{\dot{z}}$ is a genuine GWA.

6.4. The case of $h^{n}$ for $n>1$. Assume now that $f(h)=h^{n}$ for some $n>1$. In this case we have one-dimensional $\mathcal{H}(f)_{\dot{z}}$-modules with support $\mathbf{m}_{0}$. If $\dot{z} \neq 0$, we have one $\mathbb{C}^{*}$-indexed family of onedimensional simple weight $\mathcal{H}(f)_{\dot{z}}$-modules with support $\left\{\mathbf{m}_{0}\right\}$ (defined similarly as in Subsection [6.2). If $\dot{z}=0$, we have two $\mathbb{C}^{*}$-indexed families of one-dimensional simple weight $\mathcal{H}(f)_{\dot{z}}$-modules with support $\left\{\mathbf{m}_{0}\right\}$, again see Subsection 6.2 for details.

As $\mathbb{C}$ is algebraically closed, each $\mathbf{m}_{\chi}$ appears in some $\underline{\mathbf{m}} \in{ }_{\infty} Q_{\infty}$. The graph $\Phi$ has the connected component $\{0\}$ which is considered above. Another isolated component is the set of all roots of unity. This is exactly the isolated component in which each path eventually converges to an oriented cycle, in particular, it contains a lot of periodic elements in ${ }_{\infty} Q_{\infty}$. Non-periodic paths in this connected component give either double infinite string modules (if this path does not cross $\dot{z}$ ) or left- or right-infinite string modules if the path crosses $\dot{z}$. Periodic paths give band modules (again, one family of band modules with one dimensional weight spaces of the path does not cross $\dot{z}$, and two families if the path crosses $\dot{z}$ ). band modules are finite dimensional.

Finally, we have the isolated component consisting of non-zero non roots of unity. Similarly to the above this component gives double infinite string modules or left- or right-infinite string modules.

As $t$ has degree one, bounded string modules do not exist. 
Example 32. Here is an explicit example of a simple weight module which has both non-trivial finite dimensional and infinite dimensional weight spaces. Take $f(h)=h^{2}$. Define $\theta_{i}=1$ for all $i \leqslant 0$ and also define $\theta_{j}=\exp \left(\frac{2 \pi \dot{i}}{2^{j}}\right)$ for $j \geqslant 1$ (here $i$ denotes the imaginary unit). Note that $\theta_{j}^{2}=\theta_{j-1}$ for all $j \in \mathbb{Z}$. Let $\dot{z} \in \mathbb{C}$ be such that $\dot{z}+\theta_{i} \neq 0$ for all $i$. Finally, set

$$
\underline{\mathbf{m}}=\left(\ldots, \mathbf{m}_{\theta_{-1}}, \mathbf{m}_{\theta_{0}}, \mathbf{m}_{\theta_{1}}, \mathbf{m}_{\theta_{2}}, \ldots\right) .
$$

Then the $\mathcal{H}(f)_{\dot{z}}$-module $N(\underline{\mathrm{m}})$ is simple, its weight space $N(\underline{\mathrm{m}})_{\mathbf{m}_{1}}$ is infinite dimensional while all other nonzero weight spaces have dimension one.

\section{REFERENCES}

[Ba1] V. Bavula. Finite-dimensionality of $\mathrm{Ext}^{n}$ and $\mathrm{Tor}_{n}$ of simple modules over a class of algebras. (Russian) Funktsional. Anal. i Prilozhen. 25 (1991), no. $3,80-82$.

[Ba2] V. Bavula. Generalized Weyl algebras and their representations. (Russian) Algebra i Analiz 4 (1992), no. 1, 75-97.

[BB] V. Bavula, V. Bekkert. Indecomposable representations of generalized Weyl algebras. Comm. Algebra 28 (2000), no. 11, 5067-5100.

[BJ] V. Bavula, D. Jordan. Isomorphism problems and groups of automorphisms for generalized Weyl algebras. Trans. Amer. Math. Soc. 353 (2001), no. 2, 769-794.

[BO] G. Benkart, M. Ondrus. Whittaker modules for generalized Weyl algebras. Represent. Theory 13 (2009), 141-164.

[BR] G. Benkart, T. Roby. Down-up algebras. J. Algebra 209 (1998), no. 1, 305-344.

[CRM] E. Curado, M. Rego-Monteiro. Multi-parametric deformed Heisenberg algebras: a route to complexity. J. Phys. A 34 (2001), no. 15, 3253-3264.

[DGO] Yu. Drozd, B. Guzner, S. Ovsienko. Weight modules over generalized Weyl algebras. J. Algebra 184 (1996), no. 2, 491-504.

[FGM] V. Futorny, D. Grantcharov, V. Mazorchuk. Weight modules over infinite dimensional Weyl algebras. Preprint arXiv:1207.5780.

[FH1] V. Futorny, J. Hartwig. Multiparameter twisted Weyl algebras. J. Algebra 357 (2012), 69-93.

[FH2] V. Futorny, J. Hartwig. On the consistency of twisted generalized Weyl algebras. Proc. Amer. Math. Soc. 140 (2012), no. 10, 3349-3363.

[Ha1] J. Hartwig. Locally finite simple weight modules over twisted generalized Weyl algebras. J. Algebra 303 (2006), no. 1, 42-76.

[Ha2] J. Hartwig. Pseudo-unitarizable weight modules over generalized Weyl algebras. J. Pure Appl. Algebra 215 (2011), no. 10, 2352-2377.

[LZ] R. Lü, K. Zhao. Finite-dimensional simple modules over generalized Heisenberg algebras. Preprint arXiv:1401.0700

[Maz] V. Mazorchuk. A note on centralizers in $q$-deformed Heisenberg algebras. AMA Algebra Montp. Announc. 2001, Paper 2, 6 pp. (electronic).

[MT1] V. Mazorchuk, L. Turowska. Simple weight modules over twisted generalized Weyl algebras. Comm. Algebra 27 (1999), no. 6, 2613-2625. 
[MT2] V. Mazorchuk, L. Turowska. Involutions on generalized Weyl algebras preserving the principal grading. Rep. Math. Phys. 48 (2001), no. 3, 343-351.

[MT3] V. Mazorchuk, L. Turowska. *-representations of twisted generalized Weyl constructions. Algebr. Represent. Theory 5 (2002), no. 2, 163-186.

[Sh] I. Shipman. Generalized Weyl algebras: category $\mathcal{O}$ and graded Morita equivalence. J. Algebra 323 (2010), no. 9, 2449-2468.

[St] E. Steinitz. Algebraische Theorie der Körper. Chelsea Publishing Co., New York, N. Y., 1950.

R.L.: Department of Mathematics, Soochow university, Suzhou 215006, Jiangsu, P. R. China; e-mail: rencail@amss.ac.cn

V.M.: Department of Mathematics, Uppsala University, Box 480, SE751 06, Uppsala, Sweden; e-mail: mazor@math.uu.se

K.Z.: Department of Mathematics, Wilfrid Laurier University, Waterloo, Ontario, N2L 3C5, Canada; and College of Mathematics and Information Science, Hebei Normal (Teachers) University, Shijiazhuang 050016, Hebei, P. R. China. e-mail: kzhao@wlu.ca 